\title{
Hot-Band-Absorption-Induced Anti-Stokes Fluorescence of Aggregation-Induced Emission Dots and the Influence on the Nonlinear Optical Effect
}

\author{
Yuhuang Zhang ${ }^{1,+}{ }^{\dagger}$ Jing Zhou ${ }^{1,+}$, Shiyi Peng ${ }^{1}$, Wenbin Yu ${ }^{1}$, Xiaoxiao Fan ${ }^{1}$, Wen Liu ${ }^{2, *}$, Zikang Ye ${ }^{3}$, Ji Qi ${ }^{4}{ }^{\oplus}$, \\ Zhe Feng ${ }^{1}(1)$ and Jun Qian ${ }^{1, *}$
}

1 State Key Laboratory of Modern Optical Instrumentations, Centre for Optical and Electromagnetic Research, College of Optical Science and Engineering, International Research Center for Advanced Photonics, Zhejiang University, Hangzhou 310058, China; yuhuangzhang@zju.edu.cn (Y.Z.); zhoujing_bmo@zju.edu.cn (J.Z.); 22030026@zju.edu.cn (S.P.); 21630074@zju.edu.cn (W.Y.); fanxx_gs@zju.edu.cn (X.F.); zhefeng@zju.edu.cn (Z.F.)

2 Key Laboratory of Optical Information Detecting and Display Technology, Zhejiang Normal University, Jinhua 321004, China

3 Department of Chemistry, Zhejiang University, Hangzhou 310027, China; zikangye@zju.edu.cn

4 State Key Laboratory of Medicinal Chemical Biology, Key Laboratory of Bioactive Materials, Ministry of Education, College of Life Sciences, Nankai University, Tianjin 300071, China; qiji@nankai.edu.cn

* Correspondence: wenliu@zjnu.edu.cn (W.L.); qianjun@zju.edu.cn (J.Q.); Tel.: +86-187-6811-7194 (W.L.); +86-135-0581-5872 (J.Q.)

+ These authors contributed equally to this work.

Citation: Zhang, Y.; Zhou, J.; Peng, S.; Yu, W.; Fan, X.; Liu, W.; Ye, Z.; Qi, J.; Feng, Z.; Qian, J.

Hot-Band-Absorption-Induced Anti-Stokes Fluorescence of Aggregation-Induced Emission Dots and the Influence on the Nonlinear Optical Effect. Biosensors 2021, 11, 468. https://doi.org/10.3390/ bios11110468

Received: 30 September 2021 Accepted: 16 November 2021 Published: 22 November 2021

Publisher's Note: MDPI stays neutral with regard to jurisdictional claims in published maps and institutional affiliations.

Copyright: () 2021 by the authors. Licensee MDPI, Basel, Switzerland. This article is an open access article distributed under the terms and conditions of the Creative Commons Attribution (CC BY) license (https:// creativecommons.org/licenses/by/ $4.0 /)$.

\begin{abstract}
Hot-band absorption (HBA)-induced anti-Stokes fluorescence (ASF) with longer-wavelength excitation is one effective pathway to deep penetration and low autofluorescence in intravital fluorescence imaging, raising demands for fluorophores with broad spectra, high absorption, and strong emission. However, typical fluorescent dyes display some emission quenching when their concentration is increased in order to obtain brighter fluorescence. In this work, the HBA-induced ASF of aggregationinduced emission (AIE) dots is reported. BPN-BBTD dots were synthesized and confirmed with a fluorescence enhancement and a considerable ASF intensity. In addition, the mechanism of ASF and the HBA process of BPN-BBTD dots were carefully validated and discussed. To obtain the full advantages of the long-wavelength excitation and the short fluorescence lifetime in deep-tissue bioimaging, a large-depth ASF confocal microscopic imaging of in vivo cerebral vasculature was conducted under the excitation of a $980 \mathrm{~nm}$ continuous wave laser after intravenous injection of BPN-BBTD dots. Meanwhile, the $3 \mathrm{D}$ structure of the cerebrovascular network was successfully reconstructed.
\end{abstract}

Keywords: anti-Stokes fluorescence; aggregation-induced emission; hot-band absorption; in vivo confocal imaging; multi-photon microscopy

\section{Introduction}

Fluorescence imaging is a powerful tool for deep-penetration bioimaging due to its excellent resolution [1,2]. In contrast to the typical Stokes luminescence, anti-Stokes luminescence utilizes long-wavelength excitation light to emit short-wavelength photons which need additional energy. Long excitation wavelengths, such as in the second nearinfrared window (NIR-II, 900-1880 nm), are well known for their large penetration depth, low photo-damage, and low autofluorescence in biological tissues compared to visible excitation [3-10]. In addition, near-infrared excitation has lower scattering, resulting in finer focal spots and higher-quality images in deep-tissue confocal imaging [3,11,12]. Thus, these unique optical properties endow anti-Stokes luminescence with significant advantages in large-depth confocal imaging.

The phenomena of anti-Stokes luminescence can be divided into two broad categories according to different sources of additional energy $[13,14]$. One source of additional energy 
is another excitation photon, as in the multi-photon absorption process and upconversion processes based on lanthanide or triplet-triplet annihilation [5,9,15-17]. Thermal photons are another source, as in the hot-band absorption (HBA) process and the thermally activated delayed fluorescence (TADF) process $[14,18,19]$. All these processes have been successfully utilized in in vivo bioimaging, while they all have certain different limitations. The multi-photon absorption process has been widely utilized in two/three-photon microscopy. However, it commonly requires femtosecond (fs) lasers to provide high-peak-intensity excitation which is costly [20]. The bio-applications of the upconversion process are restricted by the small absorption cross-section and heavy metal ions for lanthanide-based upconversion, and the low photostability for triplet-triplet-annihilation-based upconversion [13,20-22]. The processes of thermally activated delayed fluorescence and HBA have drawn interest in recent years because they can be observed in organic dyes with high absorption $[6,14,23]$. In general, the process of anti-Stokes thermally activated delayed fluorescence is similar to the process of HBA-induced anti-Stokes fluorescence. However, the former has a long lifetime on the order of milliseconds to microseconds due to the involvement of the triplet states, in comparison with the short lifetime of the latter [14,24-28]. Because of its short fluorescence lifetime, the HBA process is suitable for fast-scanning imaging such as fluorescence confocal microscopy. Fluorescence confocal microscopy achieves high spatial resolution by placing pinholes or collimators to restrict the luminescence and detection region [29]. If fast scanning is performed, the integration time of a single pixel could be as low as $10 \mu \mathrm{s}$, which is not suitable for a long-lifetime luminescence dye [3]. However, the current organic HBA-induced anti-Stokes fluorescence dyes in use, such as indocyanine green (ICG), suffer from aggregation-induced quenching which hinders further improvement of brightness [14]. Fortunately, aggregation-induced emission (AIE) dyes were first proposed in 2001, which avoided the aggregation-caused quenching effect and emitted bright fluorescence in the aggregate or solid state [30]. When the concentration increase, fluorescence is always enhanced [31]. Thus, it could be an excellent strategy to combine AIE dyes with the HBA process in bioimaging.

In this work, the anti-Stokes fluorescence based on the HBA process is reported in AIE dyes. We prove that BPN-BBTD dyes have typical AIE characteristics, considerable anti-Stokes fluorescence intensity, and excellent photostability. By measuring the power dependence, lifetime, and temperature dependence of anti-Stokes fluorescence, the antiStokes fluorescence process was carefully determined to be the HBA process. Furthermore, considering the two absorption peaks at $363 \mathrm{~nm}$ and $706 \mathrm{~nm}$ of BPN-BBTD dyes, BPN-BBTD may have both single-photon absorption and two-photon absorption with femtosecond laser excitation. To explore the competitive relationship between HBA and multi-photon absorption, power dependence measurements of BPN-BBTD dots were performed and we found that the nonlinear effect might be weakened due to the increased HBA. In addition, to exert the long-wavelength excitation and short fluorescence lifetime in deeptissue bioimaging, anti-Stokes fluorescence confocal microscopy imaging of the cerebral vasculature in a mouse model was conducted with the largest depth of $\sim 450 \mu \mathrm{m}$ below the skull under $980 \mathrm{~nm}$ excitation. The 3D structure of the cerebrovascular network was successfully reconstructed. We believe that our work can provide a reference for future research about HBA-induced anti-Stokes fluorescence confocal microscopy and nonlinear microscopy in deep tissue.

\section{Materials and Methods}

\subsection{Chemicals and Materials}

Chloroform, Pluronic F127, and tetrahydrofuran (THF) were purchased from SigmaAldrich. Deionized water was used in all experiments. BPN-BBTD was synthesized in our laboratory as per the procedure in our previous publication [32]. 


\subsection{Fabrication of $B P N-B B T D$ Dots}

Briefly, $3 \mathrm{mg}$ BPN-BBTD in $3 \mathrm{~mL}$ chloroform was blended with $36 \mathrm{mg}$ Pluronic F-127 in $3 \mathrm{~mL}$ chloroform and sonicated for $5 \mathrm{~min}$ to get a uniform solution. Then, the mixture was evaporated in a rotating round-bottom flask until dry under vacuum at room temperature. Next, $1.5 \mathrm{~mL}$ deionized water was added to the residue and sonicated for $5 \mathrm{~min}$ until the residue was dissolved completely and formed a clear aqueous dispersion. Finally, the aqueous dispersion of BPN-BBTD dots was purified with a $0.45 \mu \mathrm{m}$ syringe filter.

\subsection{Absorption and Fluorescence Spectra Measurement}

The absorption and fluorescence spectra of BPN-BBTD dots were measured respectively by a UV-Visible spectrophotometer (measurement wavelength range 190-900 nm, UV-2550, Shimadzu, Japan) and a lab-built system for the measurement of fluorescence spectra based on a spectrometer (PG2000, Ideaoptics, Shanghai, China). To measure the Stokes fluorescence spectra, a $665 \mathrm{~nm}$ continuous-wave (CW) semiconductor laser was used as the excitation light and a $700 \mathrm{~nm}$ short-pass filter (FESH0700, Thorlabs, Newton, NJ, USA) was placed in the path of the excitation light to filter out the long-wavelength wing. The excitation beam was focused on the aqueous dispersion of BPN-BBTD dots in a quartz cell through a lens. Of note, to reduce the self-absorption of the Stokes fluorescence, the excitation light was focused near the border of the quartz cell. The Stokes fluorescence was collected by an objective $(25 \times / 1.05$, Olympus, Japan) and a $700 \mathrm{~nm}$ long-pass filter (FELH0700, Thorlabs, Newton, NJ, USA), and finally detected by the spectrometer. To measure the anti-Stokes fluorescence spectra, we replaced the $665 \mathrm{~nm}$ laser, the $700 \mathrm{~nm}$ short-pass filter, and the $700 \mathrm{~nm}$ long-pass filter with a $980 \mathrm{~nm}$ laser, a $900 \mathrm{~nm}$ long-pass filter (FELH0900, Thorlabs, Newton, NJ, USA), and a $900 \mathrm{~nm}$ short-pass filter (FESH0900, Thorlabs, Newton, NJ, USA), respectively. In addition, the quartz cell was placed on a thermostatic table to evaluate fluorescence spectra at different temperatures.

\subsection{Animal Preparation for Cerebrovascular Microscopic Imaging}

Institute of Cancer Research (ICR) mice (female, 6 weeks old) were used for in vivo experiments. They were provided by the Zhejiang Academy of Medical Sciences and raised at the Experimental Animal Center of Zhejiang University. The room temperature of the rearing environment was maintained at $24{ }^{\circ} \mathrm{C}$ with a $12 \mathrm{~h}$ light/dark cycle. Mice were continuously supplied with water and standard laboratory chows. All the animal procedures were conducted in accordance with "The National Regulation of China for Care and Use of Laboratory Animals" and supported by the Institutional Ethical Committee of Animal Experimentation of Zhejiang University. After anesthesia, the skull of the mouse was partly removed via microsurgery. Next, the hole in the skull was covered with a round thin glass to protect the brain, and a small metal ring was attached to the skull with dental cement. The mouse was fixed on a mouse rack to stabilize its head. Then, the aqueous solution of BPN-BBTD dots $(2 \mathrm{mg} / \mathrm{mL}, 200 \mu \mathrm{L})$ was intravenously injected.

\subsection{Optical Setup of the First Near-Infrared (NIR-I, 760-900 nm) Anti-Stokes Fluorescence Confocal Microscopy}

Briefly, a $980 \mathrm{~nm}$ continuous-wave semiconductor laser was collimated and reflected by a $900 \mathrm{~nm}$ short-pass dichroic mirror (DMSP900R, Thorlabs, Newton, NJ, USA), and introduced into a 2-axis ( $\mathrm{X}$ and $\mathrm{Y}$ ) scanning galvanometer system, which deflected the beam to scan. Then, the excitation light passed through the scan lens, tube lens, and objective lens $(25 \times / 1.05$, Olympus, Japan), and was finally focused on the sample. The anti-Stokes fluorescence emitted by the sample was collected by the same objective and passed back through the same optical path. The anti-Stokes fluorescence then passed through the $900 \mathrm{~nm}$ short-pass dichroic mirror, a $700 \mathrm{~nm}$ long-pass filter, and a $900 \mathrm{~nm}$ short-pass filter to remove the excitation light. A collimator was set to couple the anti-Stokes fluorescence into a fiber as a pinhole (core diameter $=1 \mu \mathrm{m}$ ). The optical signal propagated along the 
fiber to the detection plane of a PMT (H7422-50, Hamamatsu, Japan). Current signals were converted into digital signals to reconstruct images on the computer.

\subsection{Power Dependence Measurement at Different Temperatures}

In this measurement, we used a lab-built measuring system to explore the relationship between the anti-Stokes fluorescence intensity, excitation power, and temperature. Samples in the quartz cell were heated and kept at different temperatures with a thermostat. A thermal imager (TiS20, Fluke, Everett, WA, USA) was applied to accurately monitor the temperature of samples. A tunable femtosecond laser $(80 \mathrm{MHz}$, Coherent Chameleon Ti: Sapphire, USA) and a tunable continuous-wavelength laser (Matisse, Spectra-Physics, Milpitas, CA, USA) were utilized to provide excitation wavelengths at $980 \mathrm{~nm}$. The collimated excitation beam was reflected by a $900 \mathrm{~nm}$ short-pass dichroic mirror and focused via a high-numerical-aperture objective $(25 \times / 1.05$, Olympus, Japan) on the samples. The anti-Stokes fluorescence signals were collected by the same objective and passed through the same dichroic mirror to remove excitation photons. After passing through a $700 \mathrm{~nm}$ long-pass filter and a $900 \mathrm{~nm}$ short-pass filter, the anti-Stokes fluorescence was focused by a lens and detected by the PMT in sequence. An amplifier (C12419, Hamamatsu, Japan) was used to convert current signals generated by the PMT to voltage signals. Finally, a NI data acquisition card (USB-6008, National Instruments, Austin, TX, USA) sampled voltage signals for further calculation. The order of fluorescence intensity is obtained by linear fitting after logarithm of excitation power and fluorescence intensity, which reflects the proportion of linear and nonlinear components in fluorescence. When exploring the influence of temperature on power dependence, the power dependence at each temperature was measured three times independently, and the mean and standard deviation were calculated.

\subsection{Anti-Stokes Fluorescence Lifetime Measurement}

The anti-Stokes fluorescence lifetime was measured via a time-correlated singlephoton counting (TCSPC) system. A $980 \mathrm{~nm}$ femtosecond pulsed laser beam was introduced into an inverted microscope. The inside optical path was similar to Section 2.5. Finally, the anti-Stokes fluorescence signals were extracted and detected by an avalanche photodiode ( $\tau$-SPAD, PICOQUANT, Germany). The computer with an integrated TCSPC module (DPC-230 Photon Correlator, Becker \& Hickl GmbH, Berlin, Germany) was used to record the fluorescence lifetime of samples based on the synchronous signals output by the femtosecond laser and electrical signals from the $\tau$-SPAD. The fluorescence lifetime is equal to the time when the fluorescence intensity decreases from the peak to one of $1 / \mathrm{e}(\mathrm{e}$ is the base of the natural logarithm).

\subsection{Optical Setup for Photobleaching Test}

A $980 \mathrm{~nm}$ excitation light from a continuous-wave semiconductor laser was collimated and then expanded by a lens. In addition, ground glass was introduced to uniformly illuminate the flat cuvette containing BNP-BBTD dots. The anti-Stokes fluorescence was collected by a prime lens (focal length $=35 \mathrm{~mm}$, Tekwin, China) and passed through a $700 \mathrm{~nm}$ long-pass filter and a $900 \mathrm{~nm}$ short-pass filter. After that, a wide spectral responsive Si-based camera (GA1280, Tekwin, China) was set to detect the anti-Stokes fluorescence.

\section{Results and Discussion}

\subsection{Characterizations of $B P N-B B T D$}

As reported in our previous work [32], BPN-BBTD is an AIE dye with bright nearinfrared fluorescence. The molecular structure is presented in Figure 1A. The twisting phenyl/naphthyl rings restrict the intramolecular motion and increase the fluorescence intensity when molecules are in the aggregate state. In order to increase the biocompatibility of the BPN-BBTD dyes, a type of amphiphilic polymer, F127, approved by the US Food and Drug Administration, was used to encapsulate dyes into hydrophilic dots (Figure 1B). 
As shown in Figure 1C, there is an overlap between the absorption and Stokes fluorescence spectra of the BPN-BBTD dots. Moreover, the BPN-BBTD had two absorption peaks at $360 \mathrm{~nm}$ and $706 \mathrm{~nm}$, and an emission peak at $924 \mathrm{~nm}$. We obtained near-infrared fluorescence images of BPN-BBTD dots in aqueous dispersion (Figure S1) using a commercial fluorophotometer as $350 \mathrm{~nm}, 400 \mathrm{~nm}, 450 \mathrm{~nm}$, and $500 \mathrm{~nm}$ light sources, which further verified that it did have absorption at short wavelength. The small Stokes shift of BPN-BBTD dots held the possibility of thermally activated delayed fluorescence or HBA-induced anti-Stokes fluorescence. Then, a $980 \mathrm{~nm}$ continuous-wave laser was chosen instead of the $665 \mathrm{~nm}$ continuous-wave laser to excite the BPN-BBTD dots. As shown in Figure 1D, the anti-Stokes fluorescence spectrum between 700 and $900 \mathrm{~nm}$ was recorded.

Then, the AIE property of anti-Stokes fluorescence was investigated by changing the proportion of water in the THF/water mixture. As shown in Figure 1E, the anti-Stokes fluorescence intensity initially decreased with an increase in the proportion of water in the $\mathrm{THF} /$ water mixture. When the fraction of water exceeded $40 \%$, the anti-Stokes fluorescence intensity increased with the increase of water fraction. This indicated that the anti-Stokes fluorescence of BPN-BBTD dots had AIE properties under the excitation of the continuouswave laser. The photostability of BPN-BBTD dots in water was further evaluated by a continuous $980 \mathrm{~nm}$ laser irradiation for $30 \mathrm{~min}$ with a relatively large power density of $\sim 500 \mathrm{~mW} / \mathrm{cm}^{2}$. It was worth noting that the anti-Stokes fluorescence intensity negligibly reduced during irradiation (Figure $1 \mathrm{~F}$ ).

\subsection{Mechanism of Anti-Stokes Fluorescence in BPN-BBTD Dots and Its Effect on Nonlinear Optics}

There are four typical energy-conversion mechanisms to generate anti-Stokes fluorescence, according to the literature [32]. The multi-photon absorption (MPA) process is shown in Figure 2A, in which process molecules simultaneously absorbed two or more low-energy photons to emit one high-energy photon. A typical mechanism of the lanthanide-doped upconversion process is shown in Figure $2 \mathrm{~B}$, in which lanthanide ions absorb two or more low-energy photons in sequence to emit one high-energy photon. On one hand, both multiphoton absorption and upconversion processes present a power dependence value greater than one $[21,31,33,34]$. On the other hand, in thermally activated delayed fluorescence (Figure 2C) or HBA (Figure 2D) processes, one low-energy photon and additional heat energy are absorbed to emit one high-energy photon, indicating the linear power dependence of anti-Stokes fluorescence intensity. To explore the mechanism of anti-Stokes fluorescence of BPN-BBTD dots, we measured the power and temperature dependences, as well as the lifetime of anti-Stokes fluorescence. A system for measuring power dependence and spectra with a temperature controller was built for later experiments, as shown in Figure 2E. Under the excitation of a $980 \mathrm{~nm}$ continuous-wave laser, the anti-Stokes fluorescence intensity of BPN-BBTD dots versus the excitation power density was recorded in a logarithmic plot (Figure 2F) in a linearly dependent manner. As shown in Figure 2G, the lifetime of anti-Stokes fluorescence was calculated to be about $1.12 \mathrm{~ns}$. The anti-Stokes fluorescence spectra of BPN-BBTD dots were measured at different temperatures (Figure $2 \mathrm{H}$ ), and the peak intensities of spectra at different temperatures are recorded in Figure 2I. It could be observed that the anti-Stokes fluorescence intensity significantly increased along with the increase of temperature from $303.15 \mathrm{~K}$ to $353.15 \mathrm{~K}$, and the same trend occurred in the second measurement (Figure S2). The relationship between anti-Stokes fluorescence and temperature can be explained by the Boltzmann distribution:

$$
\frac{n_{i}}{n_{0}}=e^{-E_{i} / k_{B} T}
$$

where $n_{0}$ and $n_{i}$ are the molecular population of the lowest vibrational energy level and higher vibrational energy level $E_{i}$ in the ground state, respectively. $k_{B}$ is the Boltzmann constant, and $T$ is the absolute temperature. As the temperature increases, more molecules are in $E_{i}$, and fewer are in the lowest energy level of the ground state, which enhances the 
HBA-induced anti-Stokes fluorescence $[14,35,36]$. According to the above, we concluded that the anti-Stokes fluorescence of BPN-BBTD dots under the excitation of the $980 \mathrm{~nm}$ continuous-wave laser was induced by the HBA process.

A

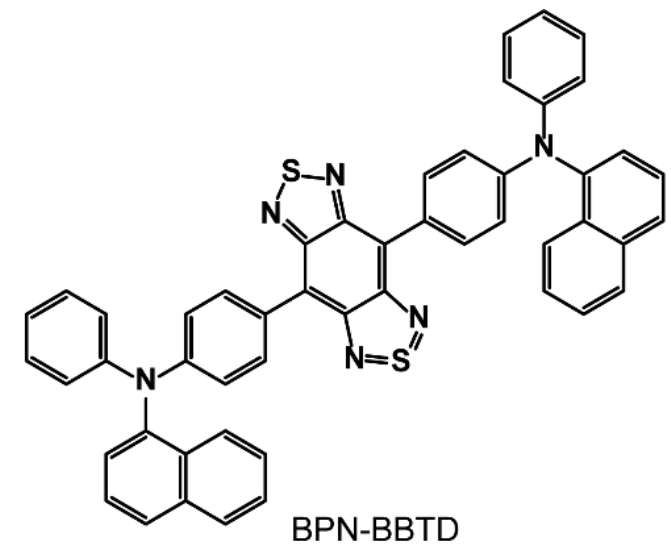

C

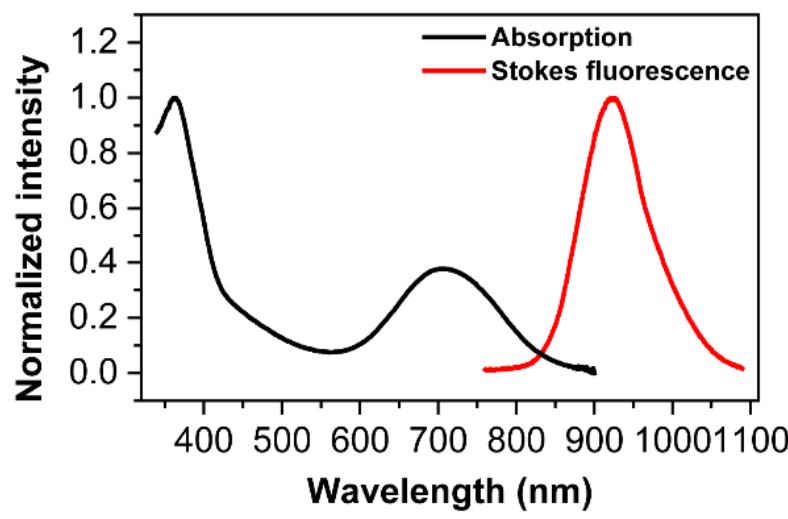

$E$



B

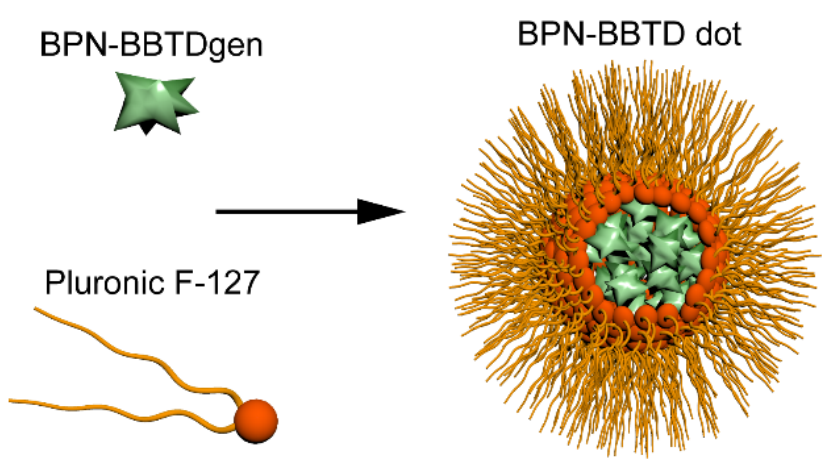

$\mathrm{F}$
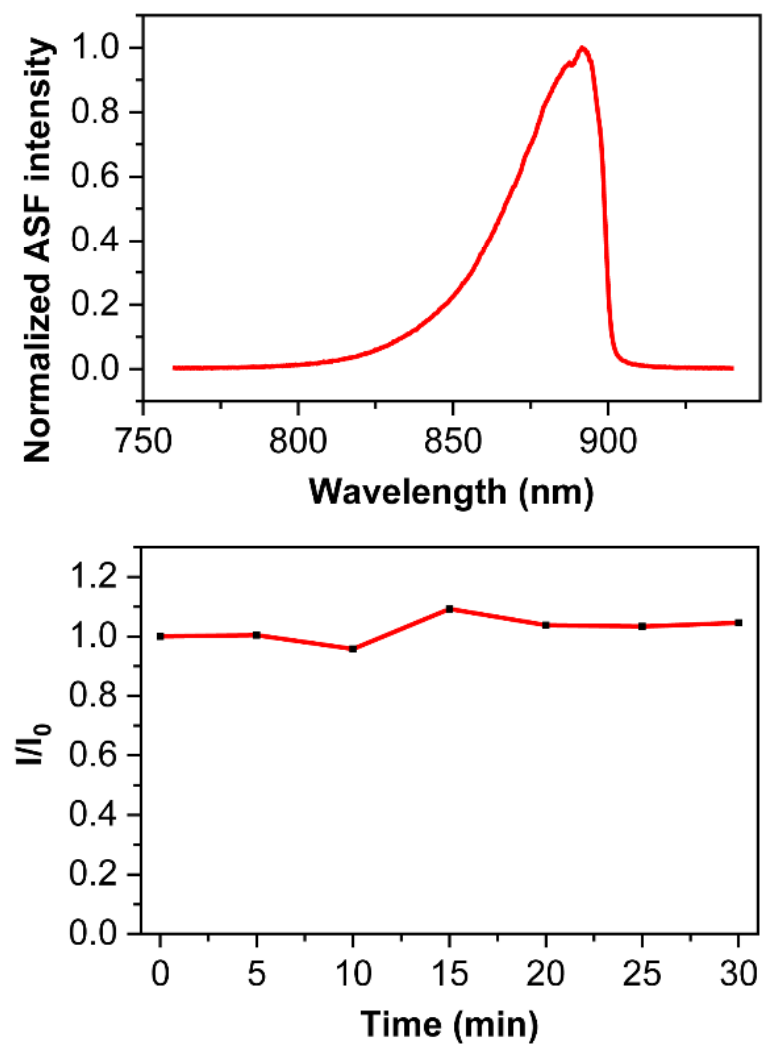

Figure 1. Characterizations of BPN-BBTD. (A) The chemical structure of the BPN-BBTD molecule. (B) The schematic illustration of the modification of BPN-BBTD dot. (C) The normalized absorption and the Stokes fluorescence spectra. The excitation wavelength for Stokes fluorescence is $665 \mathrm{~nm}$. (D) The normalized anti-Stokes fluorescence spectrum of BPN-BBTD dots in an aqueous dispersion excited by a $980 \mathrm{~nm}$ continuous-wave laser. (E) The anti-Stokes fluorescence intensity of BPN-BBTD versus the water fraction in the THF/water mixture. $\mathrm{I}_{0}$ and I are the anti-Stokes fluorescence intensities of BPN-BBTD molecules in pure THF and THF/water mixtures with specific water fractions, respectively. The concentration of BPN-BBTD is $2 \times 10^{-5} \mathrm{M}$. The excitation wavelength is $980 \mathrm{~nm}$. (F) The anti-Stokes fluorescence intensity of BPN-BBTD dots $(2 \mathrm{mg} / \mathrm{mL})$ under the continuous laser irradiation $\left(980 \mathrm{~nm}, \sim 500 \mathrm{~mW} / \mathrm{cm}^{2}\right)$. $\mathrm{I}_{0}$ and I are the anti-Stokes fluorescence intensities of BPN-BBTD dots under zero irradiation and after a certain time of irradiation, respectively. 


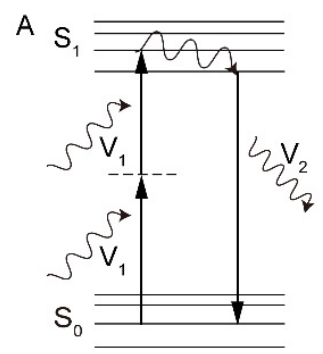

B

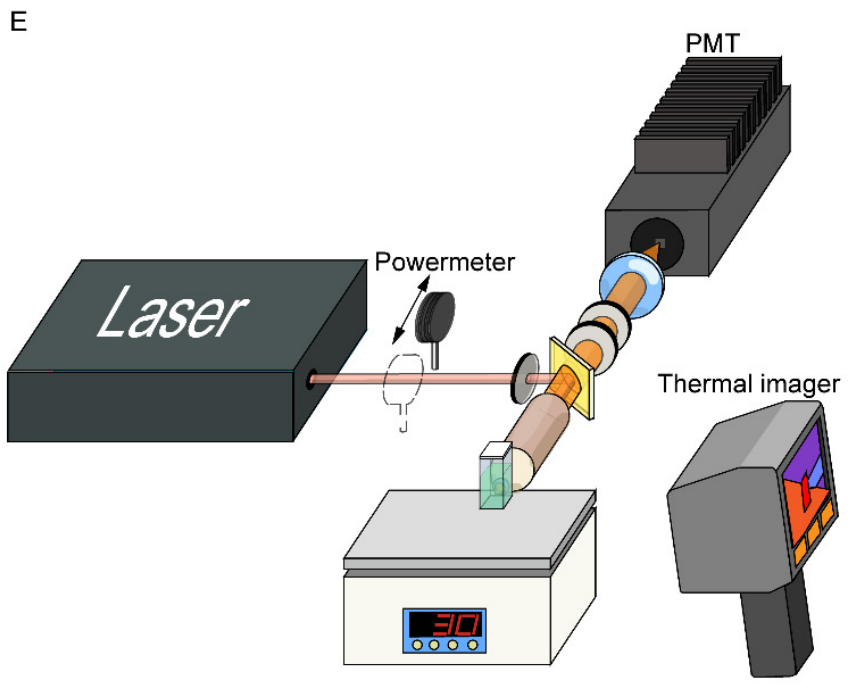

C

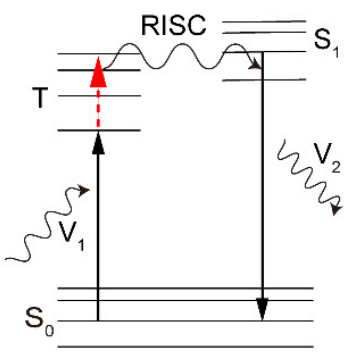

D

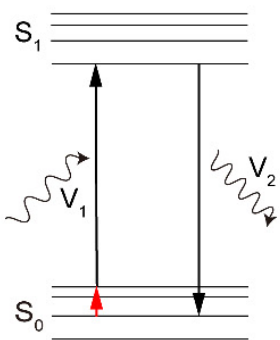

$\mathrm{F}$

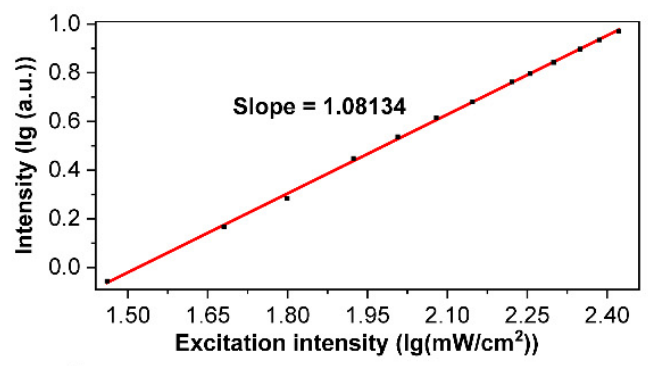

G
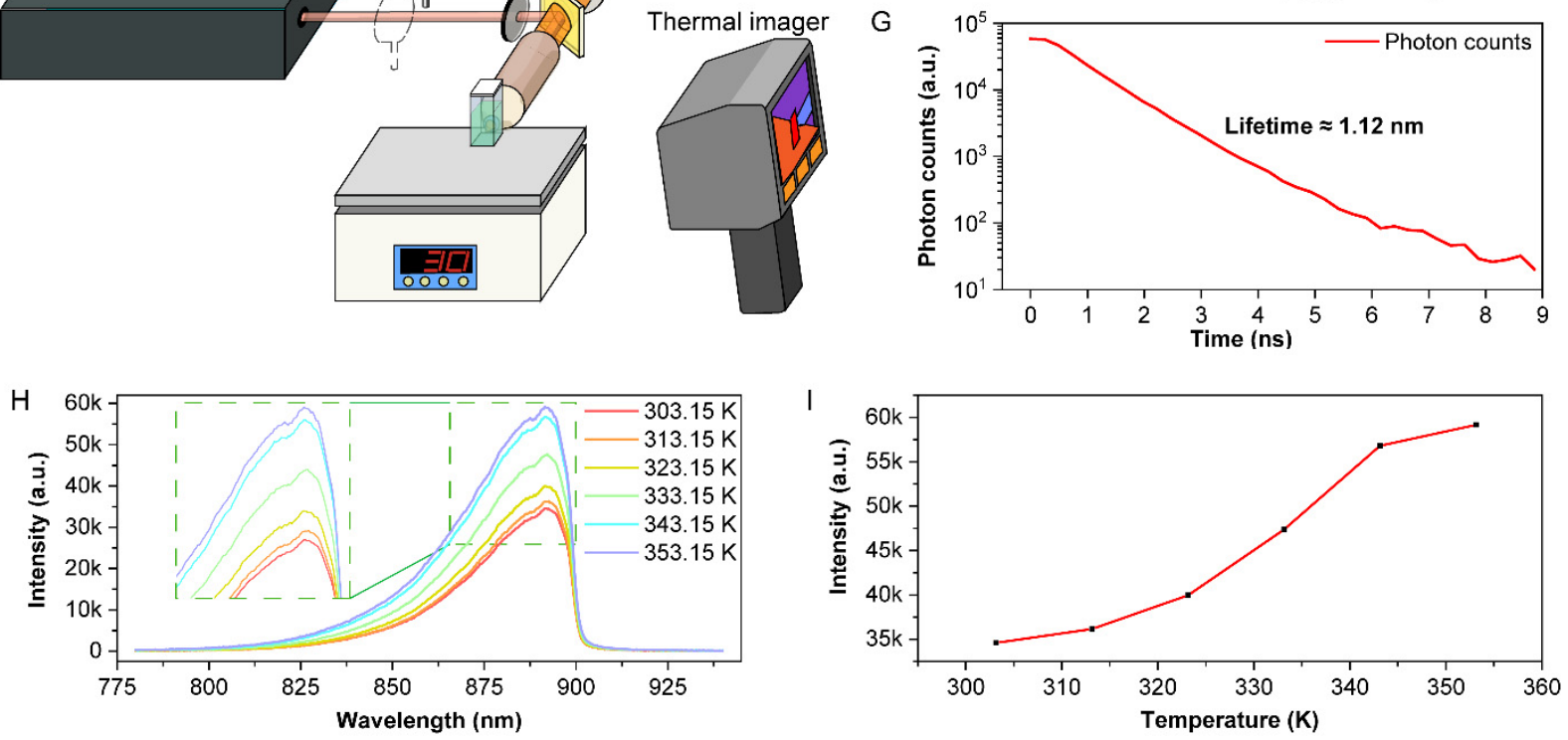

Figure 2. Mechanism of anti-Stokes fluorescence in BPN-BBTD dots. (A-D) Schematic illustrations of typical anti-Stokes fluorescence processes. (A) Two-photon absorption process. (B) Upconversion process based on multi-step absorption through intermediate energy levels. (C) Thermally activated delayed fluorescence process. (D) HBA process. (E) Optical setup for the power dependence measurement at various temperatures. (F) The logarithmic plot of anti-Stokes fluorescence intensity versus excitation light power ( $980 \mathrm{~nm}$ continuous-wave laser). The black squares and the solid line show the raw data and the fitted curve, respectively, showing a linear dependence of slope 1.08134. (G) The photon counts were plotted as a function of time under the $980 \mathrm{~nm}$ fs excitation, the lifetime of anti-Stokes fluorescence was about $1.12 \mathrm{~ns}$. (H) anti-Stokes fluorescence spectra of BPN-BBTD dots in an aqueous dispersion at different temperatures. (I) The variation of anti-Stokes fluorescence peak intensity at different temperatures under a $980 \mathrm{~nm}$ excitation.

Considering the non-negligible absorption of BPN-BBTD dots between $350 \mathrm{~nm}$ and $600 \mathrm{~nm}$ (Figure 1C), it is possible that BPN-BBTD dots could absorb two $980 \mathrm{~nm}$ photons at the same time (Figure $3 \mathrm{~A}$ ). Although the power dependence values were always about 1 , even as the temperature rose from $306 \mathrm{~K}$ to $341 \mathrm{~K}$ (Figure 3B), reconfirming that BPN-BBTD dots absorbed only one photon under the excitation of a $980 \mathrm{~nm}$ continuous-wave laser. The femtosecond laser could compress photons in time to greatly improve the excitation photon density, which promoted the two-photon absorption (2PA) process. To further investigate the relationship between HBA and two-photon absorption, the $980 \mathrm{~nm}$ femtosecond laser was chosen to excite BPN-BBTD dots. The power dependence values exceeded 1.10 at $302 \mathrm{~K}$ (Figure 3C), which indicated that there might be some two-photon absorption 

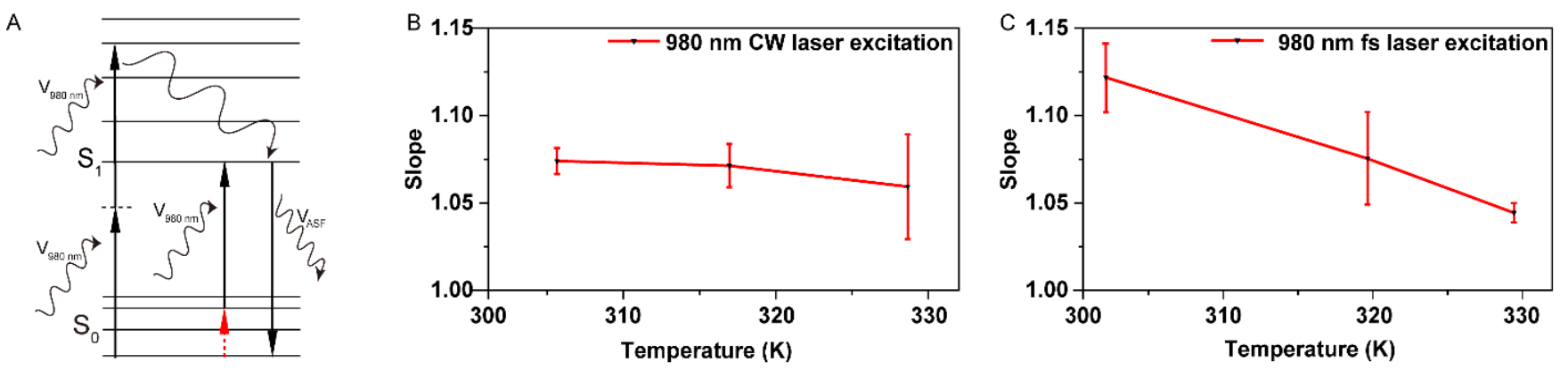

Figure 3. Effect of HBA-induced anti-Stokes fluorescence on nonlinear optics. (A) Schematic illustrations of hot-band and two-photon absorption processes of BPN-BBTD dots. The anti-Stokes fluorescence could be produced by both processes. (B) The temperature and excitation light power dependence of anti-Stokes fluorescence intensity under the excitation of continuous-wave lasers. (C) The temperature and excitation power dependence of anti-Stokes fluorescence intensity under the excitation of femtosecond lasers. Results are presented as mean $\pm \mathrm{SEM}, n=3$.

\subsection{In Vivo Anti-Stokes Fluorescence Confocal Microscopic Imaging}

To investigate the application of anti-Stokes fluorescence in bioimaging, we used BPNBBTD dots in bioimaging via a lab-built confocal microscopic system in mice. The imaging system was specially customized to utilize $980 \mathrm{~nm}$ excitation and collect $700-900 \mathrm{~nm}$ emission fluorescence. Several mice with exposed brains were imaged on the system. Figure $4 \mathrm{~A}-\mathrm{L}$ demonstrates some of the imaging results at different depths. The anti-Stokes fluorescence confocal imaging had an excellent sectioning ability, and cerebral vessels could still be distinguished even at a depth of $450 \mu \mathrm{m}$. In addition, we analyzed the signal-to-background ratios (SBRs), and the full widths at half-maximum (FWHMs) of the selected vessels, which are marked in yellow lines in the images of $220 \mu \mathrm{m}$ and $380 \mu \mathrm{m}$ depth. As shown in Figure $4 \mathrm{M}, \mathrm{N}$, the SBRs were measured to be as high as 7.25 and 1.64 , while the FWHMs were calculated to be $6.6 \mu \mathrm{m}$ and $8.1 \mu \mathrm{m}$ at those two depths, respectively. Meanwhile, we managed to reconstruct the 3D structure of the cerebral vascular network (Figure 4O). 

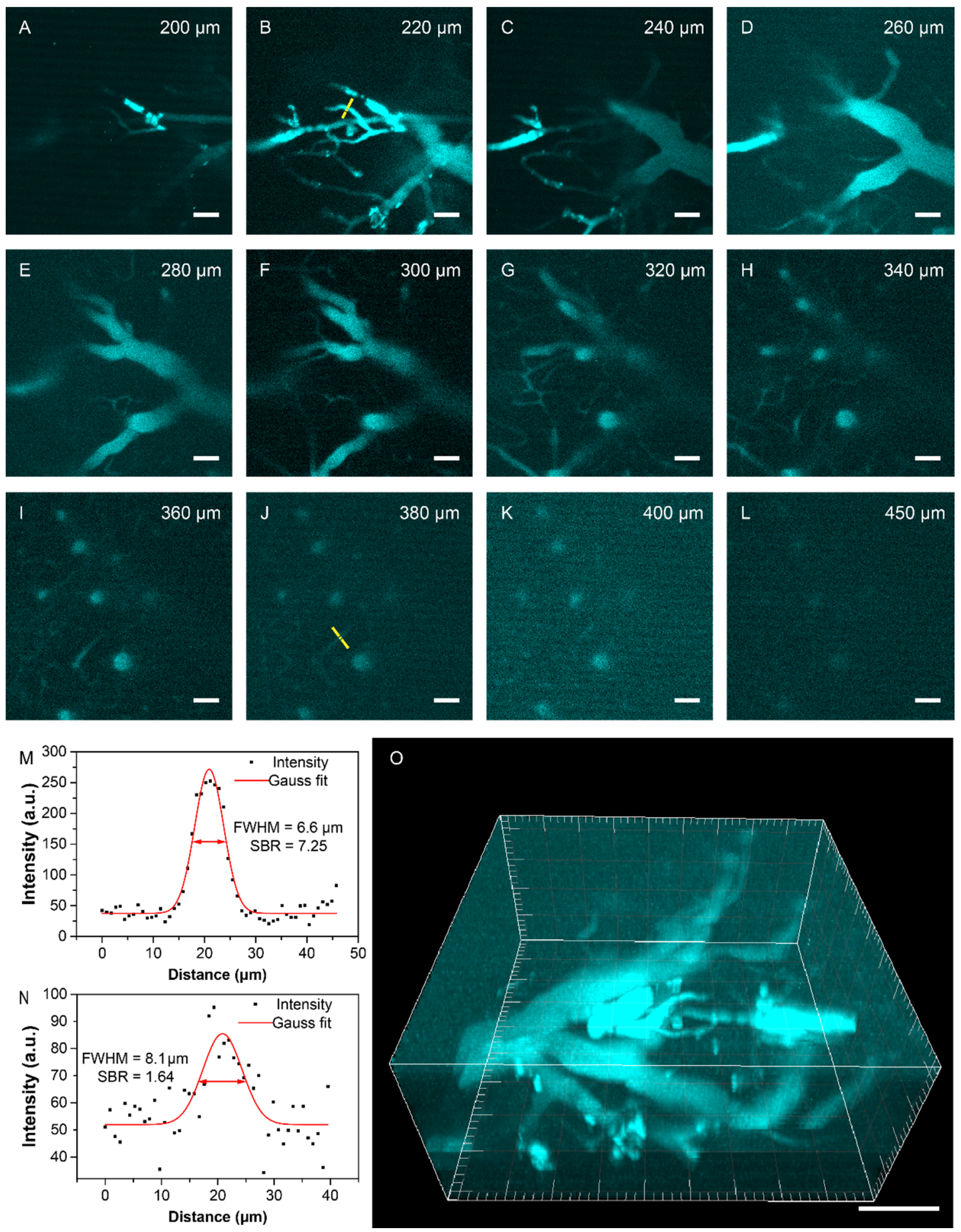

Figure 4. In vivo anti-Stokes fluorescence confocal microscopic imaging. (A-L) In vivo anti-Stokes fluorescence confocal microscopic images of brain blood vessels at various depths following the injection of BPN-BBTD dots $(2 \mathrm{mg} / \mathrm{mL}, 200 \mu \mathrm{L})$ under $980 \mathrm{~nm}$ continuous-wave laser excitation. Scale bar: $50 \mu \mathrm{m}$. (M,N) are the intensity distributions along the yellow lines in (B,J), respectively. The Gauss fits are shown in red curves. $(\mathbf{O})$ The 3D reconstruction of a mouse's cerebral vascular network with $450 \mu \mathrm{m}$ depth. Scale bar: $100 \mu \mathrm{m}$.

\section{Conclusions}

Due to the long-wavelength excitation and low autofluorescence, anti-Stokes fluorescence can be effectively utilized in deep-penetration bioimaging. Among five mechanisms of anti-Stokes fluorescence, the HBA-induced process is ignored though it has the advan- 
tages of requiring no heavy-metal ions, a short luminescence lifetime, and a high excitation efficiency under a moderate continuous-wave laser. However, the aggregation-induced quenching of common dyes limits the application of HBA-induced anti-Stokes fluorescence in bioimaging. In contrast, AIE dyes have the advantages of bright fluorescence and high photostability when encapsulated into dots, which is suitable for in vivo applications. In this work, the AIE dye BPN-BBTD was proved to have bright anti-Stokes fluorescence. The linear power dependence and the short fluorescence lifetime indicate that the anti-Stokes fluorescence of BPN-BBTD dots is based on the HBA-process under $980 \mathrm{~nm}$ continuouswave laser excitation. Additionally, the two-photon absorption process under the $980 \mathrm{~nm}$ femtosecond laser excitation could be weakened due to the increased HBA effect as temperatures increased. Additionally, the anti-Stokes fluorescence confocal microscopic imaging of mice's cerebral vasculatures was conducted under a $980 \mathrm{~nm}$ excitation. With the largest depth of $450 \mu \mathrm{m}$, the vasculature network was successfully reconstructed. To our best knowledge, this is the first report of HBA-induced anti-Stokes fluorescence with AIE properties and its application in intravital confocal microscopic imaging with a large depth. We believe that our work provides novel insight into HBA-induced anti-Stokes fluorescence confocal imaging for future research.

Supplementary Materials: The following are available online at https: / www.mdpi.com/article / 10.3390/bios11110468/s1: Figure S1: Stokes fluorescence macroscopic images of BPN-BBTD dots $(25 \mu \mathrm{g} / \mathrm{mL})$ aqueous solution at different excitation wavelengths; Figure S2: The second measured anti-Stokes fluorescence spectra of BPN-BBTD dots aqueous solution at different temperatures.

Author Contributions: Y.Z. and J.Z. contributed equally to this work. All experiments execution, Y.Z. and J.Z.; optical system preparation, Z.F., W.Y., S.P. and Z.Y.; animal preparation, Z.F. and X.F.; dyes preparation, J.Q. (Ji Qi); writing—original draft preparation, Y.Z.; writing—review and editing, W.L., J.Z. and Z.F.; funding acquisition, J.Q. (Ji Qi), W.L. and J.Q. (Jun Qian); supervision, J.Q. (Jun Qian) and W.L. All authors have read and agreed to the published version of the manuscript.

Funding: This work was supported by the National Natural Science Foundation of China (61975172, 82001874, and 82172081), the Fundamental Research Funds for the Central Universities (2020-KYY511108-0007), the Natural Science Foundation of Zhejiang Province (LR17F050001), the Zhejiang Provincial Natural Science Foundation of China (LQ21F050007), and the Open Fund of Guangdong Provincial Key Laboratory of Luminescence from Molecular Aggregate, South China University of Technology, China (2019B030301003).

Institutional Review Board Statement: The study was conducted according to the National Institutes of Health Guidelines, and approved by the Ethics Committee of Animal Use and Care Committee at Zhejiang University (ZJU20210234).

Informed Consent Statement: Not applicable.

Data Availability Statement: Not applicable.

Acknowledgments: The authors thank Yiyin Zhang for polishing the writing, and Liang Zhu for his technical assistance on the femtosecond laser.

Conflicts of Interest: The authors declare no conflict of interest.

\section{References}

1. Feng, Z.; Bai, S.; Qi, J.; Sun, C.; Zhang, Y.; Yu, X.; Ni, H.; Wu, D.; Fan, X.; Xue, D.; et al. Biologically Excretable Aggregation-Induced Emission Dots for Visualizing Through the Marmosets Intravitally: Horizons in Future Clinical Nanomedicine. Adv. Mater. 2021, 33, 2008123. [CrossRef] [PubMed]

2. Fan, X.; Xia, Q.; Zhang, Y.; Li, Y.; Feng, Z.; Zhou, J.; Qi, J.; Tang, B.Z.; Qian, J.; Lin, H. Aggregation-Induced Emission (AIE) Nanoparticles-Assisted NIR-II Fluorescence Imaging-Guided Diagnosis and Surgery for Inflammatory Bowel Disease (IBD). Adv. Healthc. Mater. 2021, 2101043. [CrossRef] [PubMed]

3. Yu, W.; Guo, B.; Zhang, H.; Zhou, J.; Yu, X.; Zhu, L.; Xue, D.; Liu, W.; Sun, X.; Qian, J. NIR-II fluorescence in vivo confocal microscopy with aggregation-induced emission dots. Sci. Bull. 2019, 64, 410-416. [CrossRef]

4. Chen, G.; Qiu, H.; Prasad, P.N.; Chen, X. Upconversion nanoparticles: Design, nanochemistry, and applications in theranostics. Chem. Rev. 2014, 114, 5161-5214. [CrossRef] [PubMed] 
5. Liu, Q.; Feng, W.; Yang, T.; Yi, T.; Li, F. Upconversion luminescence imaging of cells and small animals. Nat. Protoc. 2013, 8, 2033-2044. [CrossRef]

6. Liu, Y.; Su, Q.; Zou, X.; Chen, M.; Feng, W.; Shi, Y.; Li, F. Near-infrared in vivo bioimaging using a molecular upconversion probe. Chem. Commun. 2016, 52, 7466-7469. [CrossRef]

7. Guo, B.; Feng, Z.; Hu, D.; Xu, S.; Middha, E.; Pan, Y.; Liu, C.; Zheng, H.; Qian, J.; Sheng, Z.; et al. Precise Deciphering of Brain Vasculatures and Microscopic Tumors with Dual NIR-II Fluorescence and Photoacoustic Imaging. Adv. Mater. 2019, $31,1902504$. [CrossRef]

8. $\quad$ Feng, Z.; Tang, T.; Wu, T.; Yu, X.; Zhang, Y.; Wang, M.; Zheng, J.; Ying, Y.; Chen, S.; Zhou, J.; et al. Perfecting and extending the near-infrared imaging window. Light Sci. Appl. 2021, 10, 197. [CrossRef]

9. Li, D.; Zhang, H.; Streich, L.L.; Wang, Y.; Lu, P.; Wang, L.; Prevedel, R.; Qian, J. AIE-nanoparticle assisted ultra-deep three-photon microscopy in the in vivo mouse brain under $1300 \mathrm{~nm}$ excitation. Mater. Chem. Front. 2021, 5, 3201-3208. [CrossRef]

10. Yu, X.; Ying, Y.; Feng, Z.; Qi, J.; Zheng, J.; Zhang, Y.; Liu, J.; Qian, J.; Tang, B.Z.; Zhang, D. Aggregation-induced emission dots assisted non-invasive fluorescence hysterography in near-infrared IIb window. Nano Today 2021, 39, 101235. [CrossRef]

11. Yu, X.; Feng, Z.; Cai, Z.; Jiang, M.; Xue, D.; Zhu, L.; Zhang, Y.; Liu, J.; Que, B.; Yang, W.; et al. Deciphering of cerebrovasculatures via ICG-assisted NIR-II fluorescence microscopy. J. Mater. Chem. B 2019, 7, 6623-6629. [CrossRef] [PubMed]

12. Feng, Z.; Yu, X.; Jiang, M.; Zhu, L.; Zhang, Y.; Yang, W.; Xi, W.; Li, G.; Qian, J. Excretable IR-820 for in vivo NIR-II fluorescence cerebrovascular imaging and photothermal therapy of subcutaneous tumor. Theranostics 2019, 9, 5706-5719. [CrossRef] [PubMed]

13. Zhu, X.; Su, Q.; Feng, W.; Li, F. Anti-Stokes shift luminescent materials for bio-applications. Chem. Soc. Rev. 2017, 46, 1025-1039. [CrossRef] [PubMed]

14. Zhou, J.; Fan, X.; Wu, D.; Liu, J.; Zhang, Y.; Ye, Z.; Xue, D.; He, M.; Zhu, L.; Feng, Z.; et al. Hot-band absorption of indocyanine green for advanced anti-stokes fluorescence bioimaging. Light Sci. Appl. 2021, 10, 182. [CrossRef]

15. Wang, T.; Xu, C. Three-photon neuronal imaging in deep mouse brain. Optica 2020, 7, 947-960. [CrossRef]

16. Zhou, J.; Wen, S.; Liao, J.; Clarke, C.; Tawfik, S.A.; Ren, W.; Mi, C.; Wang, F.; Jin, D. Activation of the surface dark-layer to enhance upconversion in a thermal field. Nat. Photonics 2018, 12, 154-158. [CrossRef]

17. Di, X.; Wang, D.; Zhou, J.; Zhang, L.; Stenzel, M.H.; Su, Q.P.; Jin, D. Quantitatively Monitoring In Situ Mitochondrial Thermal Dynamics by Upconversion Nanoparticles. Nano Lett. 2021, 21, 1651-1658. [CrossRef]

18. Uoyama, H.; Goushi, K.; Shizu, K.; Nomura, H.; Adachi, C. Highly efficient organic light-emitting diodes from delayed fluorescence. Nature 2012, 492, 234-238. [CrossRef]

19. Fontenot, R.; Mathur, V.; Barkyoumb, J.; Mungan, C.; Tran, T. Measuring the Anti-Stokes Luminescence of CdSe/ZnS Quantum Dots for Laser Cooling Applications. Proc. SPIE 2016, 9821, 1-7. [CrossRef]

20. Larson, D.R.; Zipfel, W.R.; Williams, R.M.; Clark, S.W.; Bruchez, M.P.; Wise, F.W.; Webb, W.W. Water-Soluble Quantum Dots for Multiphoton Fluorescence Imaging in Vivo. Science 2003, 300, 1434-1436. [CrossRef]

21. Zhang, Y.; Jiang, D.; Yang, W.; Wang, D.; Zheng, H.; Du, Y.; Li, X.; Li, Q. Near-infrared-emitting colloidal Ag2S quantum dots exhibiting upconversion luminescence. Superlattices Microstruct. 2017, 102, 512-516. [CrossRef]

22. Liu, Q.; Yang, T.; Feng, W.; Li, F. Blue-Emissive Upconversion Nanoparticles for Low-Power-Excited Bioimaging in Vivo. J. Am. Chem. Soc. 2012, 134, 5390-5397. [CrossRef] [PubMed]

23. Xiong, X.; Song, F.; Wang, J.; Zhang, Y.; Xue, Y.; Sun, L.; Jiang, N.; Gao, P.; Tian, L.; Peng, X. Thermally Activated Delayed Fluorescence of Fluorescein Derivative for Time-Resolved and Confocal Fluorescence Imaging. J. Am. Chem. Soc. 2014, 136, 9590-9597. [CrossRef] [PubMed]

24. Chen, Z.; Wu, Z.; Ni, F.; Zhong, C.; Zeng, W.; Wei, D.; An, K.; Ma, D.; Yang, C. Emitters with a pyridine-3,5-dicarbonitrile core and short delayed fluorescence lifetimes of about $1.5 \mu$ s: Orange-Red TADF-based OLEDs with very slow efficiency roll-offs at high luminance. J. Mater. Chem. C 2018, 6, 6543-6548. [CrossRef]

25. Skaisgiris, R.; Serevičius, T.; Kazlauskas, K.; Geng, Y.; Adachi, C.; Juršènas, S. Origin of dual emission in $\sigma$-bridged donor-acceptor TADF compounds. J. Mater. Chem. C 2019, 7, 12601-12609. [CrossRef]

26. Yuan, J.; Tang, Y.; Xu, S.; Chen, R.; Huang, W. Purely organic optoelectronic materials with ultralong-lived excited states under ambient conditions. Sci. Bull. 2015, 60, 1631-1637. [CrossRef]

27. Chen, J.-X.; Tao, W.-W.; Chen, W.-C.; Xiao, Y.-F.; Wang, K.; Cao, C.; Yu, J.; Li, S.; Geng, F.-X.; Adachi, C.; et al. Red/Near-Infrared Thermally Activated Delayed Fluorescence OLEDs with Near $100 \%$ Internal Quantum Efficiency. Angew. Chem. Int. Ed. 2019, 58, 14660-14665. [CrossRef]

28. Redmond, R.W.; Gamlin, J.N. A Compilation of Singlet Oxygen Yields from Biologically Relevant Molecules. Photochem. Photobiol. 1999, 70, 391-475. [CrossRef]

29. Kachynski, A.V.; Kuzmin, A.N.; Pudavar, H.E.; Prasad, P.N. Three-dimensional confocal thermal imaging using anti-Stokes luminescence. Appl. Phys. Lett. 2005, 87, 023901. [CrossRef]

30. Luo, J.; Xie, Z.; Lam, J.W.Y.; Cheng, L.; Chen, H.; Qiu, C.; Kwok, H.S.; Zhan, X.; Liu, Y.; Zhu, D.; et al. Aggregation-induced emission of 1-methyl-1,2,3,4,5-pentaphenylsilole. Chem. Commun. 2001, 18, 1740-1741. [CrossRef]

31. Liu, W.; Zhang, Y.; Qi, J.; Qian, J.; Tang, B.Z. NIR-II Excitation and NIR-I Emission Based Two-photon Fluorescence Lifetime Microscopic Imaging Using Aggregation-induced Emission Dots. Chem. Res. Chin. Univ. 2021, 37, 171-176. [CrossRef] 
32. Alifu, N.; Zebibula, A.; Qi, J.; Zhang, H.; Sun, C.; Yu, X.; Xue, D.; Lam, J.W.Y.; Li, G.; Qian, J.; et al. Single-Molecular NearInfrared-II Theranostic Systems: Ultrastable Aggregation-Induced Emission Nanoparticles for Long-Term Tracing and Efficient Photothermal Therapy. ACS Nano 2018, 12, 11282-11293. [CrossRef]

33. Tian, L.; Xu, Z.; Zhao, S.; Cui, Y.; Liang, Z.; Zhang, J.; Xu, X. The Upconversion Luminescence of Er3+/Yb3+/Nd3+ Triply-Doped $\beta$-NaYF4 Nanocrystals under 808-nm Excitation. Materials 2014, 7, 7289. [CrossRef] [PubMed]

34. Han, M.; Zhu, Z.; Ouyang, M.; Liu, Y.; Shu, X. Highly Efficient Triplet-Triplet-Annihilation Upconversion Sensitized by a Thermally Activated Delayed Fluorescence Molecule in Optical Microcavities. Adv. Funct. Mater. 2021, 2104044. [CrossRef]

35. Jia, T.; Wang, Q.; Xu, M.; Yuan, W.; Feng, W.; Li, F. Highly efficient BODIPY-doped upconversion nanoparticles for deep-red luminescence bioimaging in vivo. Chem. Commun. 2021, 57, 1518-1521. [CrossRef]

36. Treanor, C.E.; Rich, J.W.; Rehm, R.G. Vibrational Relaxation of Anharmonic Oscillators with Exchange-Dominated Collisions. J. Chem. Phys. 1968, 48, 1798-1807. [CrossRef] 